\title{
Estereotipos femeninos y preferencia de consumo
}

\section{Women's Stereotypes and Consumer Preferences}

Recibido: abril 19 de 2010 | Revisado: julio 2 de 2010 | Aceptado: agosto 4 de 2010

\author{
ANDREA VELANDIA-MORALES ** \\ ROSA RODRÍGUEZ-BAILÓN \\ Universidad de Granada, España
}

Para citar este artículo. Velandia-Morales, A., \& Rodríguez-Bailón, R. (2011). Estereotipos femeninos y preferencia de consumo. Universitas Psychologica, 10(1), 47-59.

* Artículo de investigación

*** Departamento de Psicología Social y Metodología de las Ciencias del Comportamiento. Correos electrónicos: andreave@correo.ugr.es; rrbailon@ugr.es

\section{RES U MEN}

De acuerdo a la teoría del Sexismo Ambivalente (Glick \& Fiske, 1996) existen estereotipos diferenciados de mujeres, hacia los que los hombres manifiestan distintas actitudes. Entre los subtipos de mujeres destacan el ama de casa, la mujer sexi y la profesional. Mediante un estudio con 100 participantes, se investigó la relación entre estos tres tipos de mujeres, su nivel de influencia y prestigio, y la preferencia por un producto (descrito en términos estereotípicamente masculinos y femeninos). Los resultados mostraron que la mujer sexi es más asociada a la descripción masculina, mientras que la mujer profesional lo es a la descripción femenina; el ama de casa es la menos asociada a las dos descripciones del producto. Se encontró también que la influencia y el prestigio median la relación entre los tres tipos de mujeres, y la preferencia atribuida de cada una de ellas por el producto descrito en términos femeninos.

Palabras clave autores

Psicología Social, Psicología del Consumidor, sexismo ambivalente, estereotipos, influencia, prestigio, preferencia de producto.

Palabras clave descriptores

Estereotipos sexuales, sexismo, aspectos psicológicos, Psicología del Consumidor.

\section{A B S T R A C T}

According to The Ambivalent Sexism Theory (Glick y Fiske, 1996) there are distinct stereotypes of women that men express different attitudes. Among them, the housewife, sexi women and executive women are the clearest ones. One hundred people participated in the present study in order to test the relationship between the female stereotypes, their level of influence and prestige and the level of preference for a commercial product (described in female and male terms). The results showed that sexi women is more associated with the masculine description, whereas the executive women is more associated to the feminine product description, and in both cases the housewife is the least associated with the two different descriptions. It was also found that the influence and the women prestige mediated the relationship between the stereotypes and the preference shown for the product described in feminine terms.

Key words authors

Social Psychology, Consumer Psychology, Ambivalent Sexism, Stereotypes, Influence, Prestige, Product Preference.

Key words plus

Sex Stereotypes, Sexism, Psychological Aspects, Consumer Psychology. 
Investigaciones actuales evidencian que las decisiones de compra de los consumidores pueden estar mediadas por comportamientos impredecibles y azarosos (De Negri, 2001; Pinson, 1986; Quintani1la, 1997). Además, se reconoce que las decisiones de los consumidores también quedan influidas por otro tipo de factores en conjunción con los meramente cognitivos, tales como los motivacionales, sociales y emocionales (Botero, Abello, Chamorro $\&$ Torres, 2005; Gardner, 1985; Sandoval, 2006). Todo ello evidencia la necesidad de la Psicología del Consumidor que, como su nombre lo indica, estudia el comportamiento del consumidor y que, además, resulta útil en el conocimiento de la dinámica que se gesta entre el consumidor y su realidad social, donde variables como la cultura, etnocentrismo, significados simbólicos y tradiciones, entre otros, se analizan como factores que median las actitudes, intenciones de compra, y preferencias de consumo (Luque-Martínez, Ibáñez-Zapata \& Barrio-García, 2000; Páramo, 2004; Quintanilla, 2002, citados por Velandia-Morales \& LópezLópez, 2008).

\section{Estereotipos y Género}

Los estereotipos permiten percibir a los individuos como miembros de una categoría, asumiendo que tienen cualidades relevantes de ésta sin necesidad de comprobarlos (García-Marques \& Mackie, 1999). Además, pueden constituir una exageración de la realidad que en algunos casos ayuda a justificar el orden social, por lo que muchos autores los han identificado como una de las principales causas del prejuicio hacia los grupos (Tajfel, 1981; Tajfel \& Turner, 1979). Específicamente, los estereotipos de género son creencias generales acerca del sexo, asociadas a roles, características psicológicas y conductas que describen a hombres y mujeres (Alport, 1954; Fiske, 1993; Glick \& Fiske, 2001; Plakoyiannaki, Mathioudaki, Dimitratos \& Zotos, 2008). De acuerdo con la Teoría de los Roles Sociales, estos estereotipos se derivan de los comportamientos que los hombres y las mujeres típicamente desempeñan, más allá de las diferencias biológicas (Eagly \& Mladinic, 1994; Glick \& Fiske, 2001), por lo que pueden llegar a adquirir un carácter normativo y prescriptivo, que determina cuáles son los comportamientos y actitudes deseables para cada género. Así, generalmente, las mujeres han sido asociadas a roles dentro del hogar y los hombres a roles públicos (Vigorito \& Curry, 1998).

Relacionando las variables género, poder, influencia y estatus, se ha encontrado que las diferencias de poder e influencia entre hombres y mujeres pueden favorecer el conflicto en las relaciones de género y crear antipatía intergrupal e ideologías hostiles (Brauer \& Bourhis, 2006; Glick \& Fiske, 2001; Fiske \& Dépret, 1996). Respecto al estatus, se ha hallado que los roles masculinos tradicionalmente han sido considerados con más estatus que los femeninos (Camussi \& Leccarddi, 2005; Glick \& Fiske, 1996; Glick, Wilk \& Perreault, 1995).

\section{La Teoría del Sexismo Ambivalente}

Lo dicho hasta aquí da pie para introducir la Teoría de Sexismo Ambivalente de Glick y Fiske (1996), la cual propone la forma en la que las relaciones estructurales entre hombres y mujeres generan actitudes ambivalentes (hostiles y benevolentes), hacia las mujeres. Las actitudes hostiles legitiman el control de los hombres sobre las mujeres, ubicando a estas últimas como grupo inferior y subordinado. Estas actitudes negativas se expresan hacia aquellas mujeres que desafían el poder de los hombres o que no cumplen con el rol tradicional femenino. Por otro lado, el sexismo benevolente también considera a las mujeres como inferiores, limitándolas a roles tradicionalmente femeninos (madre, esposa y objeto romántico), que necesitan de un hombre que las cuide (Glick \& Fiske, 1996). Es este último el que dificulta que las mujeres se manifiesten en contra del patriarcado como filosofía, ya que dentro de él se les premia idealizándolas y protegiéndolas (si cumplen los roles tradicionales), lo que podría verse como una estrategia que permite mantener el poder de los hombres sobre las mujeres (Moya, Páez, Glick, Fernández Sedano \& Poeschl, 2001). La inconsistencia entre el sexismo benevolente y hostil, se resuelve categorizando a las mujeres en subgrupos. Así, se pueden desplegar actitudes po- 
sitivas hacia unas y negativas hacia otras al mismo tiempo (Glick \& Fiske, 1996). La clasificación más común que se ha encontrado tiene tres categorías: la primera, en relación con la familia (ama de casa, esposa, madre) como la más prescriptiva de género; la segunda es la de mujer atractiva (mujer sexi, novia), y la tercera asociada al trabajo (mujer profesional), como la menos prescriptiva (Deaux, Winton, Crowley \& Lewis, 1984; Fiske \& Neuberg, 1990).

\section{El Sexismo Ambivalente y la Psicología del Consumidor}

Incorporar todo este conocimiento a la Psicología del Consumidor resulta relevante, ya que hay cada vez un mayor interés y despliegue de las estrategias de marketing hacia las mujeres como grupo objetivo específico, por ser las que más compras de bienes y servicios realizan (Roya-Vela, Aldas-Manzano, Küster \& Vila; 2008). Pero más allá de analizar solamente las preferencias de las mujeres hacia los productos, parece importante saber en qué medida las descripciones e imágenes de los productos ofertados activan actitudes positivas o negativas hacia los estereotipos que representan, y modulan parte de la preferencia por un producto determinado.

Goffman (1976), sugiere que la publicidad participa en la construcción de la identidad de género, ya que genera mensajes acerca de las normas y valores culturales que las personas usan para definir sus roles en la sociedad (Fowles, 1996). Esta concepción abre la discusión sobre las implicaciones sociales y éticas que tiene la publicidad en el mantenimiento de los estereotipos. Así, se subraya la importancia que tiene el conocer los posibles efectos de los estereotipos en el consumo de productos, así como en la toma de conciencia que desde la publicidad se debe tener, para evitar seguir reproduciendo imágenes sexistas que mantengan la inequidad de género (Consejo Audiovisual de Andalucía, 2007; Furnham \& Mak; 1999; Garst \& Bodenhausen, 1997; Roya-Vela et al., 2008).

Lo hasta aquí revisado indica que las mujeres con roles no tradicionales pueden ser percibidas por los hombres como una amenaza, bien sea por lo que pueden lograr a través de su sexualidad (p.e. mujer sexi), o por la competencia que tienen (mujer profesional).

En la presente investigación, se pretende explorar si estas distintas percepciones de los subtipos de mujeres se reflejarán en la preferencia atribuida a cada una de ellas, por diferentes productos de consumo. Con este objetivo se presentarán dos descripciones de un mismo producto. En un caso, el producto se mostrará de forma típicamente masculina (resaltando características interesantes para los hombres); mientras que, en el otro caso, el producto se presentará con características atractivas para un público femenino. Se predice que en términos generales existirán diferencias entre la asociación de los tipos de mujer y las dos descripciones de producto (masculina vs. femenina); así, la mujer sexi y profesional se asociará más a la descripción masculinizada; mientras que el ama de casa se asociará mayormente a la descripción feminizada.

De acuerdo a la Teoría de Sexismo Ambivalente (Fiske \& Glick, 1996), los participantes hombres, para resolver la ambivalencia de sus actitudes positivas y negativas hacia las mujeres, crearán subcategorías de éstas, de manera que ello les permita desplegar actitudes positivas hacia algunas de ellas y negativas hacia otras al mismo tiempo; así, se espera que los participantes hombres asocien de manera diferencial los tres tipos de mujer a las descripciones de producto, como una de las formas disponibles, en la presente investigación, de expresar la ambivalencia de sus actitudes hacia las mujeres y crear subcategorías de éstas. Sin embargo, respecto a las mujeres participantes no se tiene una predicción clara en este sentido.

Derivado de la Teoría de Roles Sociales, los roles masculinos se consideran más importantes que los femeninos y se asocian con un mayor estatus; por tanto, se espera que existan diferencias entre la influencia y el prestigio atribuido a los tipos de mujer presentados; así, se predice que la mujer profesional y la sexi, al ser roles menos prescriptivos de género a los cuales se les quitan características femeninas y se les atribuyen masculinas, sean percibidas significativamente con 
mayor nivel de influencia y prestigio que la mujer ama de casa. Además, se espera encontrar que el nivel de prestigio e influencia medie la relación entre el tipo de mujer presentado y la preferencia atribuida por el producto.

\section{Método}

\section{Participantes}

Se encuestaron 100 personas (50 hombres y 50 mujeres), habitantes de la ciudad de Granada (España). Su edad osciló entre los 18 y los 65 años.

\section{Instrumento}

Para el desarrollo del presente estudio, se elaboraron dos descripciones de un mismo automóvil, una con características típicamente femeninas y otra con características típicamente masculinas. A los participantes se les pidió que asociaran estas dos descripciones a tres tipos de mujer (sexi, profesional y ama de casa) y que les atribuyeran a cada una su nivel de preferencia, agrado, probabilidad de compra, y recomendación del mismo. Adicionalmente, se preguntó por la influencia y el prestigio atribuido a cada tipo de mujer. Solamente en el caso de las participantes mujeres, se midió su identificación con los tres tipos de mujer y por su propia preferencia, agrado, probabilidad de compra, y recomendación de cada descripción.

La investigación se llevó a cabo con un cuestionario diseñado para tal efecto, utilizando escalas tipo Likert y diferencial semántico, con valores de 1 a 5. El cuestionario contenía los siguientes factores en el siguiente orden:

a) Tipos de Mujer: Se presentaron por medio de imágenes en blanco y negro los tres subtipos de mujer que se proponen desde la Teoría del Sexismo Ambivalente: la mujer tradicional (Ama de Casa) y las no tradicionales (Mujer profesional y Mujer atractiva sexualmente o Sexi).

b) Descripción de un producto masculinizado y feminizado. Las características relevantes para uno y otro género se determinaron de acuerdo con distintas investigaciones que han estudiado el mercado automotriz y han detectado estas características diferencialmente por género. En este sentido se ha encontrado que las mujeres le dan más importancia a atributos como la seguridad, el fácil mantenimiento, la confiabilidad y el confort; mientras que los hombres resaltan la velocidad, la potencia, y elementos de la mecánica del automóvil (Pierpaoli, 2008). Teniendo en cuenta lo anterior, se construyeron las siguientes definiciones:

1. Descripción Automóvil Feminizado: "Un coche original, práctico e ingenioso, que sobresale por sus prácticas soluciones interiores y exteriores que facilitan su mantenimiento. Con un diseño confortable y seguro, que te da la confianza que necesitas para emprender cualquier destino".

2. Descripción Automóvil Masculinizado: "Un coche que sobresale por su aspecto deportivo, sus potentes motores y su interior flexible y vanguardista. Con un diseño y estilo atlético y compacto, que permite una mayor aceleración y velocidad; manteniendo una imagen de libertad que deseas conservar".

c) Medida de Preferencia General. Se midió teniendo en cuenta los componentes de la estructura de la preferencia: cognoscitivo, afectivo y conductual (Kinnear \& Taylor, 1998; Kotler, 1995). Así, se preguntó por la asociación del producto a cada tipo de mujer, por la medida en que el producto se considera adecuado a sus necesidades, preferencia específica, agrado, probabilidad de compra y recomendación. Todas estas cuestiones se promediaron para crear la media global de Preferencia (Alfa de Cronbach $=0.89 ; .85 ; .75$ respectivamente, para la Mujer profesional, Sexi y Ama de casa).

d) Influencia (asociada a los subtipos de mujer), que se preguntó como: ¿Cuál diría usted que es el nivel de influencia que tiene cada subtipo de mujer sobre los demás? (adaptada de Fiske, Cuddy, Glick \& Xu, 2002). 
e) Prestigio (asociado a los Tipos de mujer): se definió como el reconocimiento social del grupo (Lovaglia, 1994), y se preguntó así: ¿Cómo de prestigiosa es la posición en la sociedad de este tipo de mujer? (adaptada de Fiske, Cuddy, Glick \& Xu, 2002).

\section{Resultados}

Tal como se pretendía con la manipulación, las dos descripciones del producto se asociaron diferencialmente con el género masculino y femenino. Así, la Descripción Masculina se asoció en mayor medida con los hombres, mientras que la femenina con las mujeres $(t(99)=-10.595, p<.001$; $M=1.83$ y $M=3.39$, respectivamente), a pesar de que a los participantes no se les advirtió acerca del género al que iba dirigida cada descripción. El sexo de los entrevistados no interaccionó con esta percepción, sin embargo el orden de presentación de las descripciones sí, teniendo efecto solo sobre la descripción masculina, la cual se ve más dirigida a los hombres cuando se presenta después de la Descripción Feminizada $(M=1.62)$ que cuando se presenta en primer lugar $(M=2.04)(t(98)=$ 2.786, $p<0.05)$.

Para contrastar las dos primeras hipótesis, se hizo un ANOVA de medidas repetidas, tomando como factores intraparticipantes la Descripción del Producto (masculino vs. femenino) y el Tipo de Mujer (Ama de Casa, Sexi, Profesional), y como variables entre grupos el género de los participantes, utilizando como variable dependiente la asociación atribuida del producto para cada tipo de mujer estudiado. El ANOVA realizado evidenció un efecto principal de la variable Tipo de Mujer ( $F$ $(2,192)=247.75 ; p<.001)$. Así, independiente de la descripción presentada, al Tipo de Mujer Ama de Casa, se le asoció el producto en menor medida $(M=2.27)$ que a la Mujer Profesional ( $M$ =3.62) y a la Mujer Sexi $(M=3.61)$. Pero más interesante para nuestros objetivos, los resultados mostraron también una interacción entre Tipo de Mujer y Descripción del Producto $(F(2,192)=$ 86.39; $<$ < 0.001). Tal como se muestra en la Figura 1 , mientras que a la descripción masculina se asoció más la Mujer Sexi $(M=3.88)$ que la Mujer Profesional $(M=3.48)(t(99)=3.25, p<0.005)$; la Descripción femenina se asoció significativamente en mayor medida con la Mujer Profesional $(\mathrm{M}=$ 3.76) que con la Mujer Sexi $(M=3.34), t(99)=$ $-3.55, p<0.005$. Los resultados muestran que las dos descripciones se asocian en menor medida a la Mujer Ama de Casa $(M=1.43$, asociación con descripción masculina y $M=3.1$ con la descripción femenina). Según estos resultados, y en apoyo a la primera hipótesis planteada, la Mujer Sexi y la Profesional son más asociadas a la Descripción del producto masculinizada; mientras que el Ama de Casa se relaciona más con la Descripción del Producto feminizada.

Al hacer el análisis por tipo de mujer, se encontró que la Mujer Sexi es más asociada a la Descripción Masculina que a la Femenina $(t(99)=4.185$, $p<0.001)$, la Mujer Profesional más asociada a la Descripción Femenina que a la Masculina ( $t$ (99) $=-1.999, p<0.05$ ), y la Mujer Ama de Casa claramente más asociada a la Descripción Femenina que a la Masculina $(t(99)=-13.143, p<0.001)$.

Además, el ANOVA realizado arrojó una interacción entre el sexo de los participantes y el Tipo de mujer, $(F(2,192)=3.739 ; p<0.05)$. En el caso de los hombres, asociaron diferencialmente las dos descripciones con cada tipología de mujer $(F(2,96)$ $=54.874 ; p<0.001)$. Los hombres relacionaron en mayor medida la Descripción Masculina a la Mujer Sexi $(M=3.98)$ que a la Profesional $(M=$ 3.4), $t(49)=4.136, p<0.001$. Por el contrario la Descripción Femenina la asociaron más fuertemente con la Mujer Profesional $(M=3.88)$ que con la Mujer Sexi $(M=3.18), t(49)=-4.186, p$ $<0.005$; ambos tipos de descripciones se asociaron en último lugar con la Mujer Ama de Casa, aunque los hombres la asocian más con la Descripción Femenina $(\mathrm{M}=3.00)$ que con la Masculina $(\mathrm{M}=$ $1.42), t(49)=-9.335, p<0.001$.

Por su parte, aunque para las participantes mujeres también aparece una interacción entre el Tipo de Mujer y las dos Descripciones del Producto, ( $F$ $(2,96)=37.260 ; p<. .001)$, la Descripción del Producto Masculina, se asoció en igual medida con la Mujer Sexi $(M=3.78)$ que con la Profesional 
Figura 1

Asociación de cada descrición con cada uno de los tipos de mujer

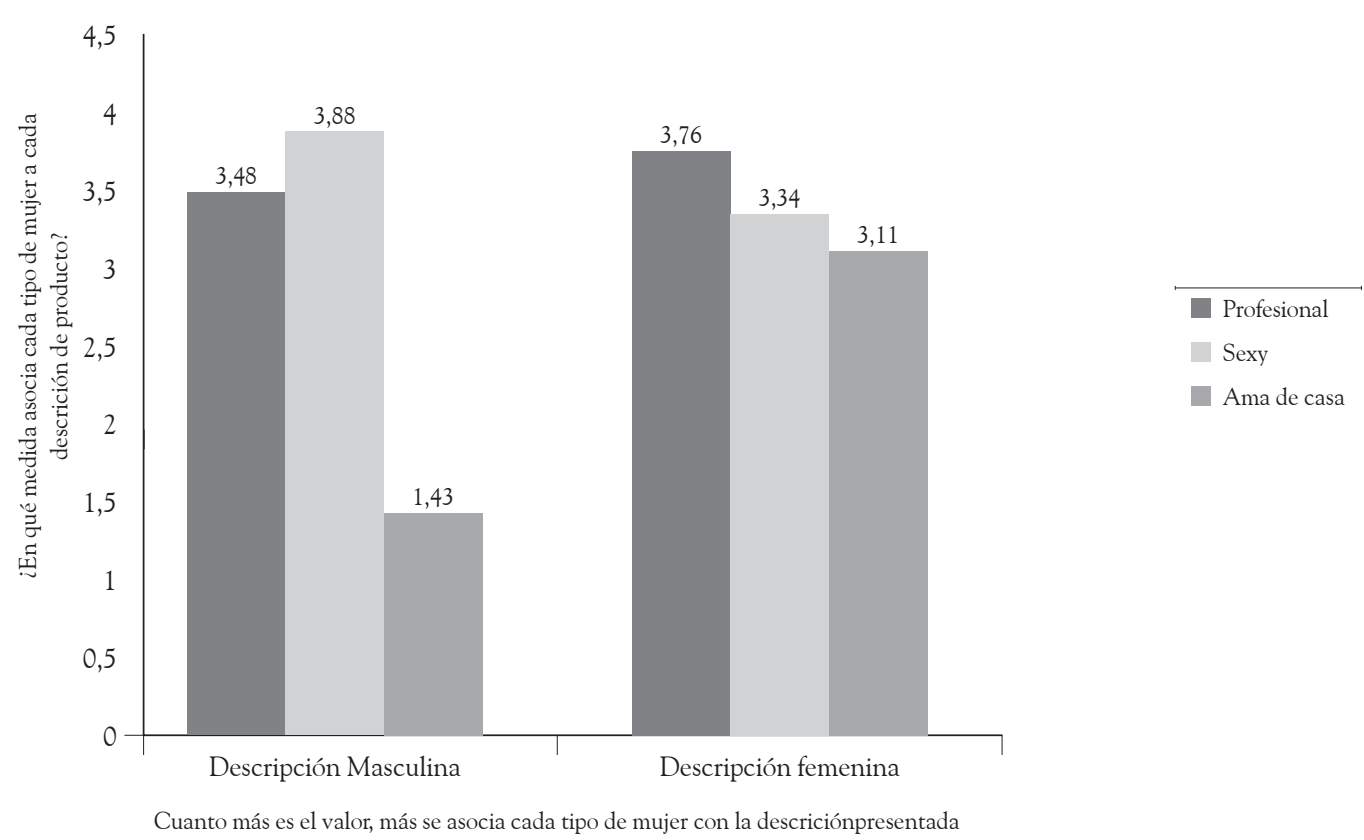

Fuente: elaboración propia.

$(M=3.56)$, pero significativamente menos con el Ama de Casa $(M=1.44)(t(49)=-9.257, p<$ 0.001). Respecto a la Descripción Femenina, se asoció en igual medida con la Mujer Profesional que la Mujer Sexi y el Ama de Casa $(M=3.64, M$ $=3.50$ y $\mathrm{M}=3.22$ respectivamente) tal como se muestra en la Figura 2.

Así, tal como se planteaba en la segunda hipótesis, los hombres asocian diferencialmente los tres Tipos de Mujer con las dos Descripciones, mientras que en las mujeres participantes solo aparecieron diferencias en la asociación de las descripciones del producto para la Mujer Ama de Casa.

Las variables relacionadas con la Preferencia General atribuida que se midieron fueron: Adecuación a las Necesidades de las Mujeres, Preferencia Específica, Agrado, Probabilidad de Compra y Recomendación atribuida.

Se realizó un ANOVA de medidas repetidas usando como variable dependiente este índice de Preferencia General, como variable intraparticipantes los tres Tipos de Mujer, y como variable entre grupos el género de los participantes. El aná- lisis arrojó un efecto del Tipo de Mujer $(F(2,196)$ $=137.611, p<0.001)$. Así, a la Mujer Sexi y a la Profesional se les atribuye la misma preferencia por ambas Descripciones $(M=3.6$, en ambas mujeres); mientras que al Ama de Casa se le atribuye la menor preferencia $(M=2.63), t(99)=4.60$, $p<0.001$.

De forma más interesante, y en relación a la Descripción Masculina del Producto, se encontró un efecto principal del Tipo de Mujer $(F(2,196)$ $=347.761, p<0.001)$. Este análisis muestra que se le atribuye una mayor preferencia de éste a la Mujer Sexi $(M=3.9)$ que a la Profesional $(M=$ 3.4) $(t(99)=5.601, p<.001)$, y a la Mujer Ama de Casa $(M=1.77), t(99)=-23.686, p<.001$, en su diferencia con la Sexi, y $t(99)=-21.257$, $p$ $<0.001$, en su diferencia con la Profesional.

Con objeto de precisar más los resultados, se estudiaron por separado las preguntas que componían el índice general de preferencia, y para comprobar si la presentación de las dos descripciones y los tres tipos de mujer, producían resultados diferenciales en cada una, se hizo un ANOVA de 
FIGURA 2

Asociación de cada descrición con cada uno de los tipos de mujer. En función del genero de los participantes

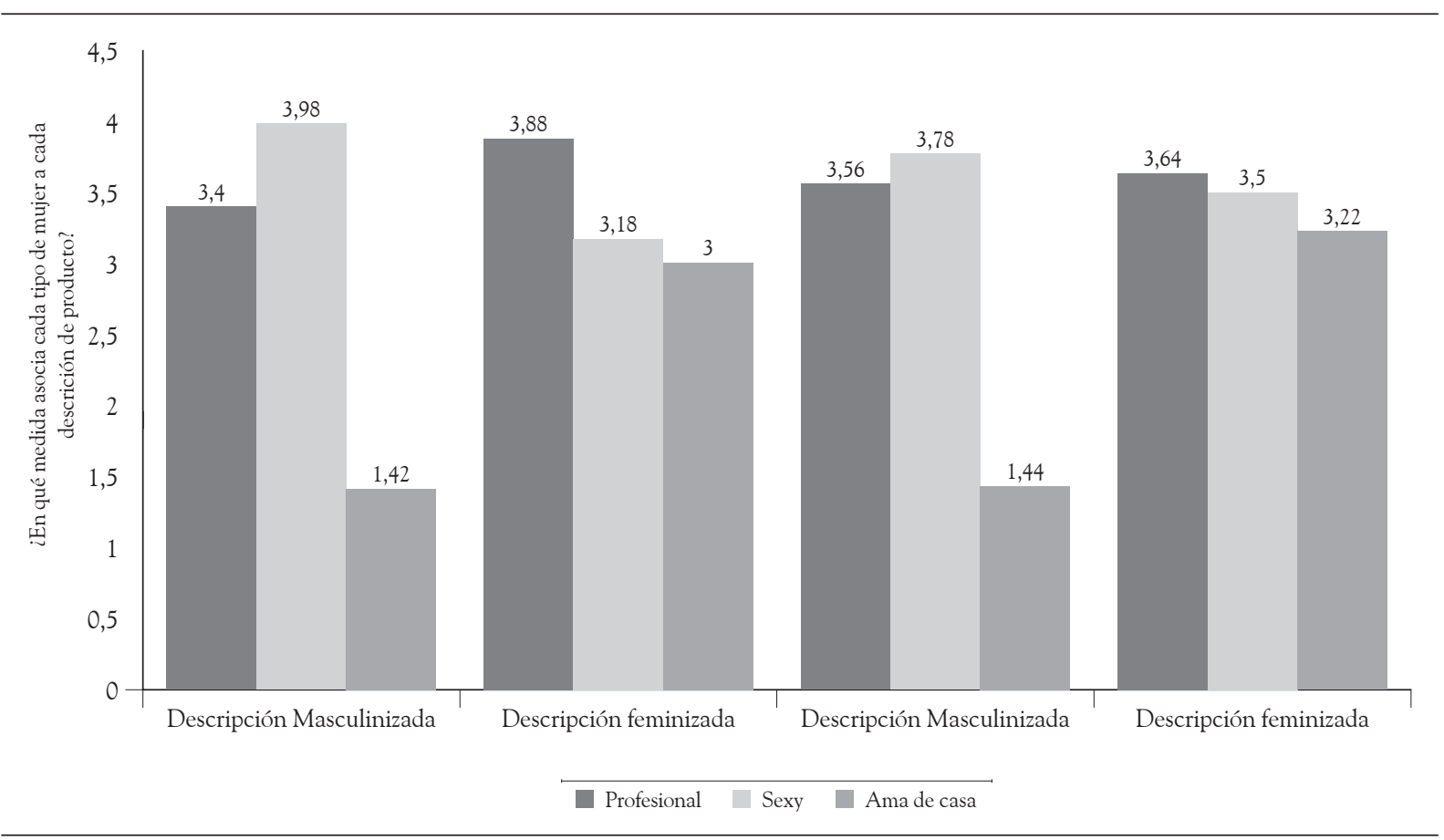

Fuente: elaboración propia.

medidas repetidas usando como variables intraparticipantes las dos Descripciones y los tres Tipos de Mujer y como variable entre grupos el género de los participantes sobre las preguntas concretas que se agruparon en el índice de preferencia general. Este resultado mostró una interacción entre los factores Descripción del Producto y Tipo de Mujer, para las siguientes medidas: Adecuación a las Necesidades de cada Tipo de Mujer $(F(2,192)=70.745$; $p<0.001)$, Preferencia Específica $(F(2,192)=$ 88.129; $p<0.001)$, Agrado atribuido ( F $(2,192)$ $=89.869 ; p<0.001)$, Probabilidad de Compra $(F$ $(2,192)=93.185 ; p<0.001)$ y Recomendación, $(F(2,192)=70.351 ; p<0.001)$; para un resumen de las medias, véase la Tabla 1.

Por último, y solo para la muestra de mujeres, se preguntó el Nivel de Identificación con cada Tipo de Mujer. Éstas se identificaron en mayor medida con la Mujer Profesional $(M=4.27)$, luego con la Mujer Sexi $(M=3.72)(t(49)=-4.328, p<$ 0.001), y por último con la Mujer Ama de Casa ( $M$ $=3.29$ ), habiendo diferencias significativas tam- bién entre estas dos últimas $(t(49)=-2.493, p<$ $.05)$ y $(t(49)=-6.708, p<0.001$ para la diferencia entre la Profesional y el Ama de Casa). Respecto a la Preferencia General personal por las Descripciones, las mujeres entrevistadas, prefirieron en mayor medida la Descripción Femenina que la Masculina $(t(49)=-4.617, p<0.001 ; M=3.81$ y $M=3.00$, respectivamente). Para determinar si existía alguna interacción entre el Nivel de Identificación con los tres Tipos de Mujer y la Preferencia General personal hacia las dos Descripciones, se realizó un ANOVA de medidas repetidas usando como medida la Preferencia General propia, como variable intraparticipantes las dos Descripciones del Producto, y como covariable la Identificación de las participantes con cada tipo de mujer, no encontrándose ninguna interacción significativa.

La hipótesis tres, predecía diferencias entre el Nivel de Influencia y Prestigio atribuido a los tres Tipos de Mujer. Así, se esperaba que la Mujer Ejecutiva y la Mujer Sexi tendrían un Nivel de Influencia y Prestigio atribuido más alto que el 


\section{TABLA 1}

Medias de adecuación de Necesidades, Preferencias Específica, Agrado, Probabilidad de Compra y recomendación atribuída para las dos Descripciones del Producto en función del Tipo de Mujer

\begin{tabular}{|c|c|c|}
\hline & Desc. Masculina Media & Desc. Femenina Media \\
\hline \multicolumn{3}{|c|}{ Se adecua a las necesidades } \\
\hline Profesional** & $3^{\mathrm{a}}$ & $4^{\mathrm{a}}$ \\
\hline Sexy & $3,51^{\mathrm{b}}$ & $3,34^{\mathrm{b}}$ \\
\hline Ama de Casa** & $1,52^{\mathrm{c}}$ & $3,64^{c}$ \\
\hline \multicolumn{3}{|c|}{ Preferencia específica } \\
\hline Profesional ${ }^{* *}$ & $3,4^{\mathrm{a}}$ & $3,93^{\mathrm{a}}$ \\
\hline Sexy** & $3,96^{\mathrm{b}}$ & $3,39^{\mathrm{b}}$ \\
\hline Ama de Casa** & $1,68^{c}$ & $3,42^{\mathrm{c}}$ \\
\hline \multicolumn{3}{|l|}{ Agrado } \\
\hline Profesional** & $3,63^{\mathrm{a}}$ & $4,94^{\mathrm{ac}}$ \\
\hline Sexy** & $4,31^{b}$ & $3,41^{\mathrm{b}}$ \\
\hline Ama de Casa* & $2,27^{\mathrm{c}}$ & $3,77^{\mathrm{c}}$ \\
\hline \multicolumn{3}{|c|}{ Probabilidad de compra } \\
\hline Profesional** & $3,53^{\mathrm{a}}$ & $3,8^{\mathrm{a}}$ \\
\hline Sexy* & $3,62^{b}$ & $3,48^{\mathrm{b}}$ \\
\hline Ama de Casa* & $1,53^{\mathrm{c}}$ & $3,52^{\mathrm{ab}}$ \\
\hline \multicolumn{3}{|l|}{ Recomendación } \\
\hline Profesional** & $3,34^{\mathrm{a}}$ & $3.84^{\mathrm{a}}$ \\
\hline Sexy** & $3,92^{b}$ & $3,29^{\mathrm{b}}$ \\
\hline Ama de Casa** & $2,2^{c}$ & $3,57^{\mathrm{c}}$ \\
\hline
\end{tabular}

* Indica diferencias significativas entre las dos descripciones del producto para cada tipo de mujer al $p<.05 ; \mathrm{y} * *<<.01$

abc Diferentes superíndices indican diferencias significativas entre los 3 Tipos de Mujer para cada una de las dos Descripciones del Producto al $p<.05$

Fuente: elaboración propia.

atribuido a la Mujer Ama de Casa. Para contrastar esta hipótesis se llevó a cabo un ANOVA de medidas repetidas, en la que se usó como medidas dependientes el Nivel de Influencia y el Prestigio, como variables intraparticipantes los tres Tipos de Mujer, y como variable manipulada entre grupos el género de los participantes. Tal como se había predicho, este análisis mostró un efecto principal de la variable Tipo de Mujer sobre el Nivel de influencia
$(F(2,196)=33.012 ; p<0.001)$ y el Prestigio $(F$ $(2,196)=107.286 ; p<0.001)$. De esta manera, a la Mujer Profesional y a la Sexi se les otorga un mayor nivel de Nivel de Influencia $(M=4.27$ y $M$ $=3.72$, respectivamente) y Prestigio $(M=4.29 \mathrm{y}$ $\mathrm{M}=3.76$, respectivamente) que a la Mujer Ama de Casa, la cual se percibe con un menor Nivel de Influencia $(M=3.29)$ y Prestigio $(M=2.62)$. 
FIGURA 3

Nivel de influencia sobre los otros y Prestigio de la posición en la sociedad de los tres tipos de mujer.

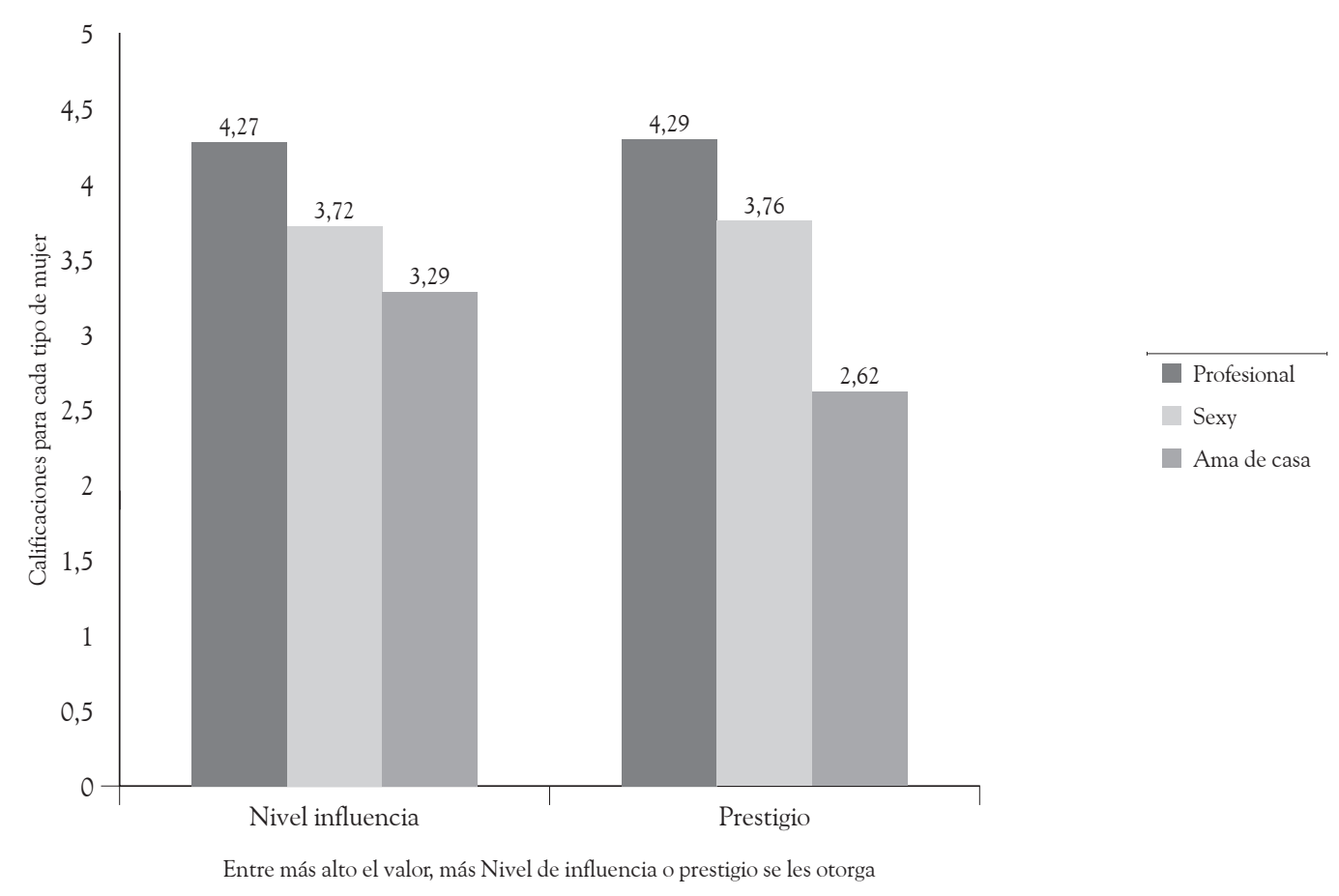

Fuente: elaboración propia.

La cuarta hipótesis proponía que el nivel de influencia y prestigio otorgado mediaría entre el Tipo de Mujer y el Nivel de Preferencia General atribuido hacia las Descripciones. Para contrastarla, se probó el efecto mediador de la influencia y el prestigio en la relación entre el Tipo de mujer y el índice de preferencia general de las descripciones. El primer análisis mediacional incluyó el Nivel de Influencia, y mostró que el Tipo de Mujer predecía significativamente el Nivel de influencia ( $\beta=$ 0.414), así como la Preferencia por la Descripción Femenina $(B=0.192)$. El Nivel de Influencia también predecía significativamente la Preferencia por esta Descripción $(B=0.296)$. Sin embargo, cuando el Nivel de Influencia y el Tipo de Mujer se introdujeron juntas en la ecuación de regresión, el Nivel de Influencia mantuvo su predicción significativa $(B=0.262)$, mientras que el Tipo de Mujer dejó de predecir la Preferencia por la Descripción Femenina $(B=0.083)$.
A continuación se realizó un análisis exactamente igual al anterior pero utilizando el prestigio como variable mediadora, encontrándose que el Tipo de Mujer predecía significativamente el Prestigio $(\beta=0.640)$ así como la Preferencia por la Descripción Femenina $(\beta=0.192)$. El Prestigio también predecía significativamente la Preferencia por la Descripción Femenina $(\beta=0.219)$. Sin embargo, cuando el Prestigio y el Tipo de Mujer se introdujeron juntas en la ecuación de regresión, aunque el Prestigio mantuvo su predicción significativa ( $(=0.163)$, el Tipo de Mujer dejó de predecir la Preferencia por la Descripción Femenina $(B=0.087)$.

Estos resultados indican que tanto el Nivel de Influencia como el Prestigio median entre el Tipo de Mujer y la Preferencia del producto descrito en términos femeninos. Por tanto, no parece ser solamente el estereotipo de los tres tipos de mujeres por sí solo el que determina la preferencia general por el producto femenino, si no el hecho de que esta 


\section{Figura 4}

Nivel de Influencia como mediador entre el Tipo de Mujer y la Preferencia del Producto Descrito de forma Femenino Los números son valores beta ( $\beta$ ). Los números en paréntesis y negrilla muestran el valor cuando la varible (que corresponda) entra sola al modelo de regresión. El valor que le sigue, es el que resulta cuando en el modelo de regresión entran la Varible Independiente y el Mediador. Niveles de significación ${ }^{* * p}<.001 ; p<.005$.

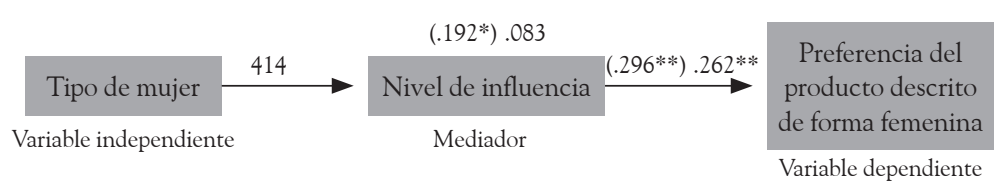

Fuente: elaboración propia.

\section{Figura 5}

Nivel de Influencia como mediador entre el Tipo de Mujer y la Preferencia del Producto Descrito de forma Femenino Los números son valores beta $(\beta)$. Los números en paréntesis y negrilla muestran el valor cuando la varible (que corresponda) entra sola al modelo de regresión. El valor que le sigue, es el que resulta cuando en el modelo de regresión entran la Varible Independiente y el Mediador. Niveles de significancia: $* * * p<.001 * * p<$ $.005 ; *<.05$.

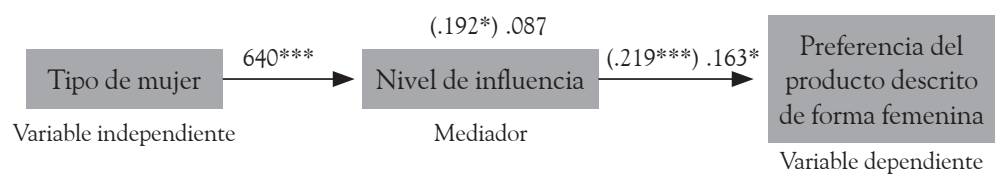

Fuente: elaboración propia.

imagen de las mujeres sexis, profesionales o amas de casa son percibidas con prestigio o influencia. Este resultado solamente aparece en el caso de la preferencia por el producto descrito en términos femeninos y no en el masculino.

\section{Discusión}

Los resultados indican que los tres Tipos de Mujer se asocian diferentemente a las dos descripciones. Comparando las dos Descripciones dentro de cada tipo de mujer, la Masculina, es claramente la más asociada a la Mujer Sexi, mientras que la Femenina a la Mujer Profesional y al Ama de Casa. Sin embargo, las mujeres entrevistadas asocian en la misma medida la descripción del producto $\mathrm{Fe}$ menina y Masculina a la Mujer Sexi y a la Mujer Profesional, diferenciando solo en el Ama de Casa, que claramente relacionan más con la Descripción femenina que con la masculina. Analizando la
Preferencia General y cada uno de sus componentes, se evidencia cómo, de nuevo, es la Mujer Sexi a la que se atribuye mayor preferencia por la Descripción Masculina, y a la Mujer Profesional por la Descripción Femenina; mientras que la Mujer Ama de Casa se mantiene con las más bajas calificaciones en ambas. También se encontró que las mujeres entrevistadas se identificaron en mayor medida con la Mujer Profesional, luego con la Mujer Sexi y por último con la Mujer Ama de Casa. En cuanto a su preferencia por las dos Descripciones del Producto, prefieren la Femenina a la Masculina, lo cual también es coherente con todas las medidas que componen el índice de Preferencia General computado.

En cuanto a influencia y prestigio atribuido, los resultados indican que a la Mujer Profesional y a la Sexi se les atribuye más influencia y prestigio que al Ama de Casa, siendo más alto para la Mujer Profesional. Por último, los análisis mediacionales 
mostraron que la influencia y el prestigio solo median la relación entre el tipo de mujer percibido y la preferencia por la descripción del producto femenina.

El hecho que las diferencias de asociación de los Tipos de Mujer con las Descripciones se deban más a los hombres que a las mujeres participantes, parecería indicar que en los primeros se activa una categoría de mujer u otra en función del rol que desempeñan (y si este es prescriptivo o no del género); mientras que en las mujeres esto ocurre en menor medida. De esta manera, los hombres asocian más a la Mujer Sexi con la Descripción Masculina, posiblemente porque la alejan de una imagen de futura madre o mujer tradicional, mientras que la Mujer Profesional, aunque es vista como competente (rasgo típicamente masculino), también se podría vincular en cierta forma a la figura de madre con un rol adicional al de trabajadora fuera del hogar. Todo ello va en concordancia con el hecho de que los estereotipos de feminidad y masculinidad se deriven de los roles que se desempeñan en el contexto social, más allá de las diferencias biológicas (Eagly \& Mladinic, 1993; Glick \& Fiske, 2001).

El hecho de que las mujeres participantes asocian más a elementos típicamente masculinos los tipos de mujer que gozan de mayor influencia y prestigio social (mujer profesional y sexi) que al "rol tradicional" de Ama de Casa, podría dejar entrever cómo las mujeres, al ser el grupo de menor estatus, utilizan elementos típicamente masculinos para aumentar, o en cierta manera equiparar, su posición frente a los hombres. Además, todo ello es perfectamente coherente con que las mujeres entrevistadas se identificaran más con la Mujer Profesional y la Sexi, pero no con el Ama de Casa. En futuras investigaciones debería profundizarse en la razón por la que, aunque las mujeres se identifican con los roles de mujeres a los mayor influencia y prestigio se les atribuye, prefieren un automóvil descrito de manera femenina, al contrario de lo que le atribuyeron a estos mismos tipos de mujeres.

A la luz de la Teoría del Sexismo Ambivalente (Glick \& Fiske, 1996), y aunque sin posibilidad de comprobación en la presente investigación puesto que no se ha medido el sexismo de los participantes, se podría esperar que el nivel de sexismo interactúe con las atribuciones de preferencia de los Tipos de Mujer. De esta manera, los hombres podrían asociar a la Mujer Profesional con la Descripción Femenina como una forma de "mantenerla en su posición de mujer relegada al hombre"; disminuyendo su competencia. Sin embargo, respecto a la Mujer Sexi, como no se le asigna el atributo de competencia, podría ocurrir que a los hombres sexistas no les importara relacionarla en mayor medida con un automóvil de tipo masculino, ya que quizás los participantes lo podrían hacer por una razón diferente. De cualquier forma, esta suposición debería probarse en investigaciones posteriores midiendo el sexismo de los participantes.

Los resultados mostrados en el presente estudio demuestran también cómo los hombres mantienen las subcategorías que hacen de las mujeres en contextos de consumo de productos.

Por otra parte, y tal como se había predicho, la Mujer Profesional y la Mujer Sexi se percibieron con un alto nivel de influencia y prestigio, no obstante estas variables solo mediaron por la preferencia hacia la Descripción del Producto Femenina y no la Masculina, dato que no parece muy coherente con la idea de que el alto nivel de influencia y prestigio podrían estar vinculados a una mayor competencia, habilidades intelectuales, y por ende a un estereotipo masculino de superioridad (Eagly \& Mladinic,1993). Por tanto, sería lógico esperar que al ser la Mujer Profesional vista con mayor influencia y prestigio, evidenciara una relación positiva entre estas variables y su asociación con la descripción masculina. Las autoras no tienen una explicación clara acerca de este resultado, pero se puede especular que podría deberse a que las mujeres quieran otorgar a los aspectos femeninos una valoración positiva y no minusvalorarlos, por lo que los asocian igualmente con influencia y prestigio. No obstante, se pone de manifiesto la necesidad de profundizar en las relaciones que subyacen entre el poder, la influencia, el prestigio y el estatus como factores mediadores del consumo de productos.

Adicionalmente, para entender mejor los resultados, sería importante replicarlos usando otras categorías de productos para determinar hasta qué 
punto la categoría elegida (un automóvil, producto claramente masculino) también tiene efecto sobre los resultados.

Este tipo de estudios y otros relacionados muestran cómo el marketing puede jugar un rol interesante en la disminución de los estereotipos. El hecho que desde la publicidad se mantengan y refuercen los estereotipos prescriptivos de los roles de género, perpetuando el sexismo y la subordinación de las mujeres frente a los hombres, indica que si se enfoca al contrario se podría ayudar a lograr la equidad de género. Además, si se hace un análisis de la publicidad en la actualidad, se evidencia un cierto tipo de incoherencia en las tácticas de marketing empleadas. Sin tener en cuenta que las mujeres se han convertido en grandes compradoras (de productos tales como casas, automóviles, productos financieros, tecnología, turismo etc.), siendo el $51 \%$ de los consumidores, y generando más del $80 \%$ del consumo, se les sigue tratando utilizando estereotipos obsoletos y peyorativos (Ganahl, Prinsen \& Netzley; 2003). Es evidente además, cómo la subcategorización que hacen los hombres de las mujeres no solo está presente en ámbitos de la vida cotidiana o el trabajo, sino en contextos de consumo de productos, por lo que debería ser una variable a tener en cuenta desde el marketing, estudiándola como una posible mediadora en los procesos de elección y compra de productos.

\section{Referencias}

Alport, G. W. (1954). The Nature of Prejudice. Reading, MA: Addison-Wesley.

Botero, M. M., Abello, L., Chamorro, M. \& Torres, V. (2005). Factores compensatorios y no compensatorios que influyen en la decisión de compra de productos culinarios en la categoría de caldos concentrados, en consumidores de la ciudad de Barranquilla. Universitas Psychologica, 4 (3), 393-402.

Brauer, M. \& Bourhis, R. (2006). Social Power. European Journal of Social Psychology 36(4), 601-616.

Camussi, E. \& Leccardy, C. (2005). Stereotypes of working women: The power of expectations [En Línea]. Social Science Information. Recuperado el
1 de agosto, 2008, de http://ssi.sagepub.com/cgi/ content/abstract/44/1/113

Consejo Audiovisual de Andalucía. (2007). Mercado y Publicidad.com [En Línea]. Andalucía, España. Recuperado el 20 de Julio de 2008, de http://www.mercadeoypublicidad.com/Secciones/Noticias/DetalleNoticias.php?recordID =9508\&pageNum busqueda $=1 \&$ totalRows_busqueda $=142 \&$ list $=\mathrm{Ok}$

De Negri, M. (2001). Introducción a la psicología económica. Revista Electrónica de Psicología, 7-28.

Deaux, K. \& Lewis, L. (1984). Structure of gender stereotypes: Interrelationships among components and gender label. Journal of Personality and Social Psychology, 46 (5), 991-1004.

Eagly, A. H. \& Mladinic, A. (1994). Are people prejudiced against women? Some answers from research on attitudes, gender stereotypes and judgments of competence. European Review of Social Psychology, $5,1-35$.

Fiske, S. T. (1993). Controlling other people: The impact of power on stereotyping. American Psychologist, 48 (6), 621-628.

Fiske, S. T., Cuddy, A. J. C., Glick, P. \& Xu, J. (2002) . A model of (often mixed) stereotype content: Competence and warmth respectively from perceived status and competition. Journal of Personality and Social Psychology, 82 (6), 878-902.

Fiske, S. \& Dépret, E. (1996). Control, interdependence and power: Understanding social cognition in its social context. Europan Review of Social Psychology, 7, 31-59.

Fiske, S. T. \& Neuberg, S. L. (1990). A continuum of impression formation, from category based to individuating processes: Influences of information and motivation on attention and interpretation. In M. P. Zanna (Ed.), Advances in Experimental Social Psychology (Vol. 23, pp. 1-74). San Diego, CA: Academic Press.

Fowles, J. (1996). Advertising and Popular Culture. Thousand Oaks, CA: Sage.

Furnham, A. \& Mak, T. (1999). Sex-role stereotyping in televisión commercials: A review and comparison of fourteen studies done in five continents over 25 years. Sex Roles, 41 (5-6), 413-437. 
Ganahl, D., Prinsen, T. \& Netzley, S. (2003). A content analysis of prime time commercials: A contextual framework of gender representation. Sex Roles, 49 (9), 545-551.

Garcia-Marques, L. \& Mackie, D. (1999). The impact of stereotype-incongruent information on perceived group variability and stereotype change. Journal of Personality and Social Psychology, 77 (5), 979-990.

Gardner, M. P. (1985). Mood states and consumer behavior: A critical review. Journal of Consumer Research, 12 (December), 281-300.

Garst, J. \& Bodenhausen, G. (1997). Advertising's effects on men's gender role attitudes. Sex Roles, 36 (9-10), 551-572.

Glick, P. \& Fiske, S. (1996). The Ambivalent Sexism Inventory: Differentiating hostile and benevolent sexism. Journal of Personality and Social Psychology, 70(3), 491-512.

Glick, P. \& Fiske, S. (2001). Ambivalent sexism. Advances in Experimental Social Psychology, 33, 115-188.

Glick, P., Wilk, K. \& Perreault, M. (1995). Images of occupations: Components of gender and status in occupational stereotypes. Sex Roles, 32 (9-10), 565-582.

Goffman, E. (1976). Gender Advertisements. New York: Harper and Row.

Kinnear, T. \& Taylor, J. (1998). Investigación de mercados: un enfoque aplicado (5aㅡ ed.). Bogotá: McGraw Hill.

Kotler, P. (1995). Manual de mercadotecnia. Sistema de información de la mercadotecnia e investigación de mercados ( $7{ }^{a}$ ed.). México: Prentice Hall.

Lovaglia, M. J. (1994). Relating power and status. Advances in Groups Processes, 11, 81-111.

Moya, M., Páez, D., Glick, P., Fernández, I. \& Poeschl, G. (2001). Sexismo, masculinidad-feminidad y factores culturales [En línea]. Reme, Revista Electrónica de Motivación y Emoción, 4 (8-9). Recuperado el 26 de Julio de 2008, de http://reme.uji.es/articulos/ amoyam 4101701102/texto.html

Páramo, D. (2004, enero-abril). El fenómeno del Consumo y el consumo en marketing. Convergencia, $34,221-250$.
Pierpaoli, A. (2008). Las mujeres siempre dan de qué hablar [En Línea]. InfoBrand. Revista de Marketing, Branding y Comunicación. Recuperado el 13 de agosto de 2008, de http://www.infobrand.com.ar/ nota.php?idx $=10629$

Pinson, C. (1986). An implicit product theory approach to consumer inferential judgments about products. International Journal of Research in Marketing, 3(1), 19-38.

Plakoyiannaki, E., Mathioudaki, K., Dimitratos, P. \& Zotos, Y. (2008). Images of women in online advertisements of global products: Does Sexism Exist? Journal of Business Ethics, 83(1), 101-112.

Quintanilla, I. (1997). Psicología económica. Madrid: MacGraw-Hill.

Quintanilla, I. (2002). Psicología del consumidor. Madrid: Pearson Educación.

Roya-Vela, M., Aldas-Manzano, J., Küster, I. \& Vila, N. (2008). Adaptation of marketing activities to cultural and social context: Gender role portrayals and sexism in Spain commercials. Sex Roles, 58(5-6), 379-390.

Sandoval, M. (2006). Los efectos de la televisión sobre el comportamiento de las audiencias jóvenes desde la perspectiva de la convergencia y de las prácticas culturales. Universitas Psychologica, 5 (2), 205-222.

Tajfel, H. (1981). Humans Groups and Social Categories. Cambridge: Cambridge University Press.

Tajfel, H. \& Turner, J. C. (1979). An integrative theory of intergroup conflict. In W. G. Austin \& S. Worchel (Eds.), The Social Psychology of Intergroup Relations (pp. 33-47). Monterey, CA: Brooks/Cole.

Velandia-Morales, A. \& López-Lopéz, W. (2008). Investigación cualitativa y psicología del consumidor. Alternativas de aplicación. Avances en Psicología Latinoamericana, 26 (2), 290-303.

Vigorito, A. \& Curry, T. (1998). Marketing masculinity: Gender identity and popular magazines. Sex Roles, $39(1-2), 135-152$. 
